\title{
A virtual test-bed for building Model Predictive Control developments
}

\author{
Raymond Sterling ${ }^{1}$, Jesús Febres ${ }^{2}$, Andrea Costa ${ }^{3}$, Adeleh Mohammadi ${ }^{1}$, Rafael E. Carrillo ${ }^{4}$, Baptiste \\ Schubnel $^{4}$, Yves Stauffer ${ }^{4}$, Pietro De Cinque ${ }^{3}$, Krzysztof Klobut ${ }^{5}$, Marcus M. Keane ${ }^{1}$ \\ ${ }^{1}$ Department of Civil Engineering, National University of Ireland, Galway, Ireland \\ \{raymond.sterling, adeleh.mohammadi, marcus.keane\}@nuigalway.ie \\ ${ }^{2}$ Fundación IK4 Tekniker, Spain, jesus.febres@tekniker.es \\ ${ }^{3}$ R2M Solution SRL, Italy, \{andrea.costa2, pietro.decinque\}@r2msolution.com \\ ${ }^{4}$ CSEM SA, PV-center, Neuchâtel, Switzerland \\ \{rafael.carrillo, baptiste.schubnel,yves.stauffer\}@csem.ch \\ ${ }^{5}$ VTT Technical Research Centre of Finland, Espoo 020400, Finland Krzysztof.Klobut@vtt .fi
}

\begin{abstract}
This paper presents the result of the work performed to develop a virtual test-bed for development and testing of Model Predictive Controllers within the district cooling networks field. These controllers are used for its application in improving the cooling energy efficiency in the network's building. The article explains the use of the different tools to develop and simulate the models with an emphasis on the advantages and challenges of co-simulation and model exchange using the Functional Mockup Interface.
\end{abstract}

Keywords: District Cooling, Modelling, FMI, Modelica, Model predictive control

\section{Introduction}

INDIGO $^{1}$ is a Horizon 2020 EU-funded project carried out by six partners from across Europe that aims to realise more efficient and economic planning, control and management of existing District Cooling (DC) networks. This will be achieved through two specific objectives. The first one is to widen the use of DC systems and motivate the competitiveness of European DC market by the development open-source tools for planning and modelling DC systems (del Hoyo Arce et al., 2018). The second objective is to reduce primary energy consumption via improved DC system management strategies aimed at system efficiency maximisation and cost minimisation.

In this paper we present the results of the work performed to improve the energy consumption of the DC systems across several tasks of the project. This includes modelling and simulation of various buildings and the development and implementation of Model Predictive Controls (MPC) to reduce energy use in buildings.

Modelling and simulation within this paper is presented for the Building models. The geometry,

\footnotetext{
${ }^{1}$ www.indigo-project.eu
}

materials, weather, air infiltration and internal gains of the models are developed in EnergyPlus and the model of the energy systems, focusing on the air distribution system while air handling units are built in Modelica.

The aim of the modelling was two-fold. To provide an accurate and validated test-bed for testing the behaviour of the MPC and, at the same time, generate the synthetic data used for the initial development of said controllers.

Model integration across different platforms is performed via Functional Mock-up Interfaces and this article presents the full workflow on the implementation from initial building model development to the generation of results from the MPC.

\section{Building Models - EnergyPlus}

In the INDIGO project, all the geometrical models of the buildings are created considering the external dimensions. This approach influences the way in which the linear transmittances of the thermal bridges are calculated. To create the model, the following information has been collected:

- Geometry of the building;

- Geometry and position of the shading objects (e.g. other buildings or trees) located around the modelled buildings;

- Distribution of the mechanical ventilation and the relative control;

- Position and properties of opaque and transparent elements (walls, roofs, windows, floors, internal partitions);

- Electrical consumption for the different buildings and for the main equipment that is installed in them, to estimate the internal gains.

For the development of the models related to the buildings, DesignBuilder v4.7.0.027 and EnergyPlus ${ }^{2}$ V8.6 were used (Figure 1). It manages input files in .idf

\footnotetext{
2 https://energyplus.net/
} 
format, which can be edited in the IDF Editor (free available online) or in a text editor. The IDF file contains both the input data that the building model acquires from the HVAC system model, which is developed in Modelica, and the output data that the building model transfers to the HVAC system model. The weather data are included in an .epw file (EnergyPlus weather file). The information included in the .idf file and that one included in the .epw file are combined in a file that is readable by Modelica, specifically in a .fmu file. The FMU is exported through a Python script (Nouidui, Wetter and Zuo, 2014).

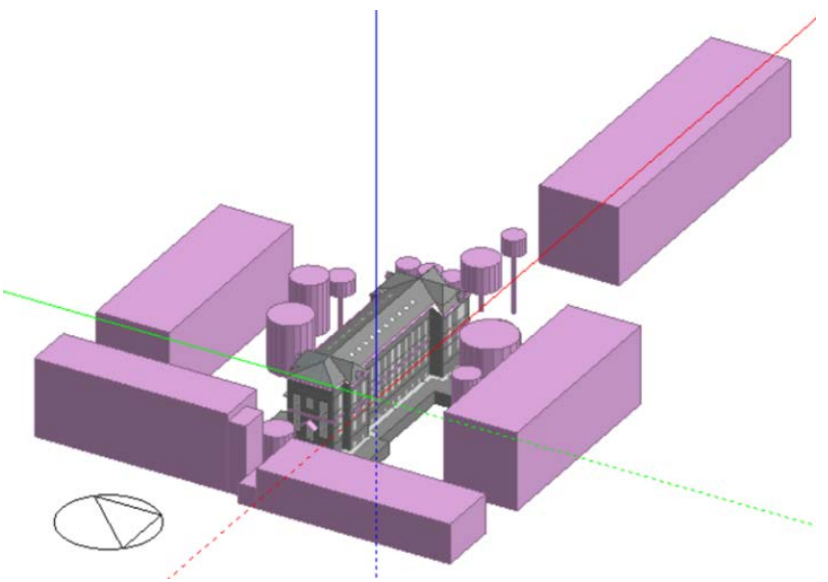

Figure 1. Aztarain model (DesignBuilder)

In the building model, there are thermal zones in which the internal air conditions are considered uniform. The thermal zones were created based on the air conditioning system and of its control logics, to allow an accurate modelling of the HVAC systems, as it is needed by the INDIGO project. As general rule, the zones whose thermal conditions are controlled by the same system (AHU) and based on the same sensors are modelled as part of the same thermal zone.

\subsection{Thermal Zones and internal gains}

For each building, the zones were created considering the following criteria t:

- Distinction between conditioned, not conditioned and specially conditioned zones (e.g. zones with special conditioning requirements);

- If two parts of the same building are served by different AHUs the two parts will be modelled separately;

- The creation of the zones considers the location of the terminal units, to define if the conditions of some rooms are controlled by a post-heating or by a postcooling coil.

- The creation of the zones also considers the location of the temperature sensors within the air distribution

\footnotetext{
${ }^{3} \mathrm{http}: / /$ www.euskalmet.euskadi.net/s07-

5853x/es/meteorologia/estacion.. apl $? \mathrm{e}=5 \mathrm{E}$ campo $=\mathrm{C} 039$
}

scheme, to model as close as possible to the reality the control logic and the temperature of the sensors on which the control logic is based.

o Rooms whose internal conditions are measured by a specific sensor (inside the room or in the return duct) are modelled separately.

o The location of the sensor is important because it defines the conditions that will be used by the control system and therefore affects the goal of the project.

- Internal gains have been modelled based on working schedules and a survey which was used to determine occupancy levels and equipment inside the zones. The occupation of the different zones was modelled considering the number of seats or beds represented in the architectural drawings.

\subsection{Weather data}

The weather data regarding dry air temperature $\left({ }^{\circ} \mathrm{C}\right)$ and relative humidity (\%) are taken on site while all the other data (solar radiation, wind velocity, wind direction, pressure) are taken from the weather station "C039 - Deusto" of the Basque agency of meteorology ("Agencia vasca de meteorología") ${ }^{3}$. The weather station is in the Bilbao city, 2,5 km away from the demo site presented in this paper.

The global radiance on a horizontal surface expressed in $\left[\mathrm{W} / \mathrm{m}^{2}\right]$ is information included in the weather file. A method provided by Reindl, D.T. et. al (1990) was used to estimate the diffuse radiation and the direct radiation from the global one. The method was validated for 5 localities in America and in Europe having very different climates (latitudes from $28.4^{\circ} \mathrm{N}$ to $59.56^{\circ} \mathrm{N}$ ) (Reindl, Beckman and Duffie, 1990).

The sun position is evaluated based on the geographical position of the building.

\subsection{Preparation for interfacing with Modelica}

To establish the communication between EnergyPlus and Modelica the use of the EnergyPlus object "ExternalInterface" is necessary. This object activates the external interface of EnergyPlus.

Currently, the only valid entries are PtolemyServer, FunctionalMockupUnitImport, FunctionalMockupUnitExport.

\subsubsection{Receiving data from Modelica}

For the INDIGO project, the option "FunctionalMockupUnitExport" was selected because the EnergyPlus file is exported as a FMU for cosimulation. The data that Modelica communicates to EnergyPlus are: 
- Sensible load [Qs] (W) due to the air supplied by the mechanical ventilation (for every zone of the building)

- Latent load [QI] (W) due to the air supplied by the mechanical ventilation (for every zone of the building)

EnergyPlus considers those loads in the same way as the internal thermal gains.

The use of heat flows instead of typical state variables (temperature, air mass flow rate, relative humidity etc.) in the data exchange from Modelica to the building FMU is motivated by modelling simplifications as exchanging state variables would significantly increase the computational effort without providing advantages over the proposed procedure.

\subsubsection{Sending Data to Modelica}

The data that EnergyPlus communicates to Modelica are:

- Temperature [T]

o Site Outdoor Air Dry-Bulb Temperature $\left({ }^{\circ} \mathrm{C}\right)$

o Zone Mean Air Temperature $\left({ }^{\circ} \mathrm{C}\right.$ ) (for every zone of the building)

- Relative humidity [RH]

o Site Outdoor Air Relative Humidity (\%)

o Zone Mean Air Relative Humidity (\%) (for every zone of the building)

- Humidity ratio [X]

o Site Outdoor Air Humidity Ratio ( $\left.\mathrm{kg}_{\text {Water }} / \mathrm{kg}_{\text {DryAir }}\right)$

o Zone Mean Air Humidity Ratio $\left(\mathrm{kg}_{\text {Water }} / \mathrm{kg}_{\text {DryAir }}\right.$ ) (for every zone of the building)

To calculate the heat flows between the HVAC system and the building, Modelica requires knowledge of the temperature and humidity conditions of the zones. Therefore, these are the variables selected to be exchanged from the FMU to Modelica.

\section{HVAC Models - Modelica}

On the demo site for the INDIGO project, six types of air handling units were identified. However, for reasons of space in this paper, this work focuses on one type which is the most common and the one described in the case study, for further information please refer to (Sterling et al., 2017).

Modelica models for HVAC systems use components based on the Modelica.Fluid library to replicate the schematic of the units. In INDIGO, all AHU will have fresh (port_F) and supply (port_S) port connections. For those units with return air, return (port_R) and exhaust (port_E) port connection are added.

All units will output the heat flow of each active component (e.g. heating coils and cooling coils).
Nominal design conditions have been imposed for the cooling coil models since no information about the input conditions on the water side of the cooling coils is being gathered by the BMS. Such conditions correspond with constant input water temperature and constant maximum mass flow rate achieved when valve is $100 \%$ open.

\subsection{AHU Model}

For this research work, a full-sized air handling unit type is demonstrated. It is composed of:

- Heat recovery (HR): two heat exchangers interconnected via a water circuit;

- Cooling Coil (CC);

- Heating Coil (HC);

- Fans;

- Humidifier (H);

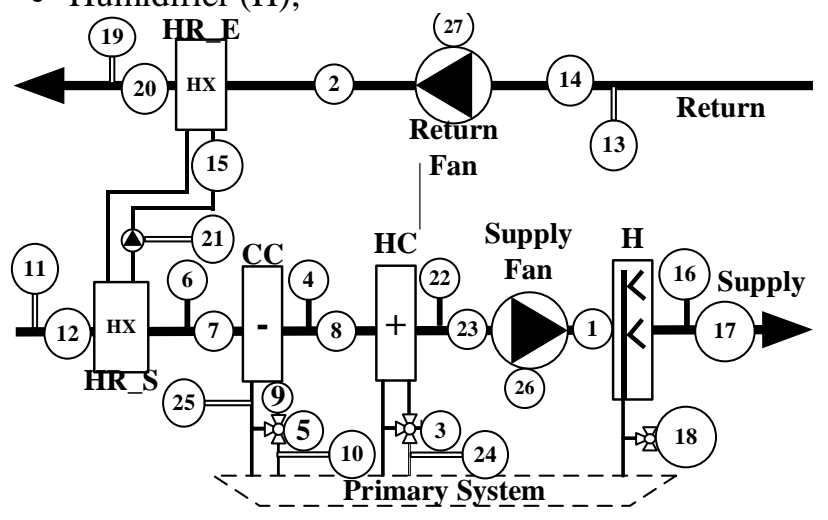

Figure 2. Schematic of the AHU under study

Table 1. AHU Model Components and Variables.

Type: M: Measurement, I: Input

\begin{tabular}{|l|l|l|l|}
\hline Component & Variable & Type & $\#$ \\
\hline Fan Supply & air mass flow rate & M & 1 \\
\hline Fan Return & air mass flow rate & M & 2 \\
\hline Heating Coil & Valve position & I & 3 \\
\hline Cooling Coil & Air output T & M & 4 \\
\hline Cooling Coil & Valve position & I & 5 \\
\hline Cooling Coil & Air input T & M & 6 \\
\hline Cooling Coil & Air input RH & M & 7 \\
\hline Cooling Coil & Air output RH & M & 8 \\
\hline Cooling Coil & Water mass flow rate & M & 9 \\
\hline Cooling Coil & Water input T & M & 10 \\
\hline HR Supply & Air input T & M & 11 \\
\hline HR Supply & Air input RH & M & 12 \\
\hline HR Exhaust & air input T & M & 13 \\
\hline HR Exhaust & Air input RH & M & 14 \\
\hline HR Exhaust & water mass flow rate & M & 15 \\
\hline Humidifier & Air output T & M & 16 \\
\hline Humidifier & Air output RH & M & 17 \\
\hline Humidifier & Valve position & I & 18 \\
\hline
\end{tabular}




\begin{tabular}{|l|l|l|l|}
\hline HR Exhaust & Air output T & M & 19 \\
\hline HR Exhaust & Air output RH & M & 20 \\
\hline HR water & Pump pressure & M & 21 \\
\hline Heating Coil & Air output T & M & 22 \\
\hline Heating Coil & Air output RH & M & 23 \\
\hline Heating Coil & Water input T & M & 24 \\
\hline Cooling Coil & Water output T & M & 25 \\
\hline Fan Supply & Pump pressure & M & 26 \\
\hline Fan Return & Pump pressure & M & 27 \\
\hline
\end{tabular}

\subsection{AHU Controllers}

The AHU has an associated control system (named AHU_controller) emulating the behaviour of the real system as closely as possible with the available data (e.g. O\&M manuals from the demo site).

The AHU_controller operates in a mode-switching hybrid system, i.e., it is a system that can operate in multiple modes, and can switch between these modes either through continuous- or discrete-valued signals. The AHU can operate in 2 nominal modes for temperature control: (1) when the controlled temperature is above its set-point plus dead-band (T_ASP) and (2) when the controlled temperature is below its set-point minus dead-band (T_BSP):

In the case of T_ASP, the AHU_controller shall:

- Modulate the opening signal valve of the Heating Coil towards the fully closed position.

- Modulate the opening signal valve of the Cooling Coil towards the fully opened position.

In the case of T_BSP, the AHU_controller shall:

- Modulate the opening signal valve of the Cooling Coil towards the fully closed position.

- Modulate the opening signal valve of the Heating Coil towards the fully opened position

All modulations are performed via PID control.

In this controller humidity control operates independently from the heating/cooling operation. For humidity control, the AHU can also operate in two nominal modes: (1) when the controlled relative humidity is above its set-point plus dead-band (RH_ASP) and (2) when the controlled relative humidity is below its set-point minus dead-band (RH_BSP).

In the case of RH_ASP, the AHU_controller shall:

- Modulate the opening signal valve of the Cooling Coil towards the fully opened position.

- Modulate the opening signal valve of the Humidifier towards the fully closed position.

In the case of RH_BSP, the AHU_controller shall:

- Modulate the opening signal valve of the Cooling Coil towards the fully closed position.

- Modulate the opening signal valve of the Humidifier Coil towards the fully opened position

All modulations are performed via PID control.

In this controller, heat recovery control operates independently from the heating/cooling operation. Heat recovery operates in on/off mode as follows:

- If (Cooling Coil valve $>0$ and $\mathrm{dH}>0$ ) or (Heating Coil valve $>0$ and $\mathrm{dH}<0$ ) then 1.0 else 0.0 .

According to maintenance personnel from the demo site, fans operate at fixed mass flow rate $100 \%$. Hence, in this controller, fan output is always true.

\section{Whole Building Model}

\subsection{FMU Interfacing}

EnergyPlus is a well-established, whole building energy simulation tool that considers a broad range of different characteristics of the buildings. It is an optimal tool to simulate the long-term (days, months and years) energy performance of the buildings. However, the implementation of the HVAC systems within EnergyPlus does not account for dynamics of diverse elements of such systems (heat exchangers, ducts, boilers, etc.) making this tool poorly accurate for shortterm (minutes and hours) simulations. To overcome this issue, we decided to integrate an EnergyPlus model of the buildings (geometry, materials, weather, internal gains) with HVAC models developed in Modelica via the Functional Mock-up Interface ${ }^{4}$. Figure 3 shows the data exchange, at each time-step, between HVAC model in Modelica and each zone in the EnergyPlus building model.

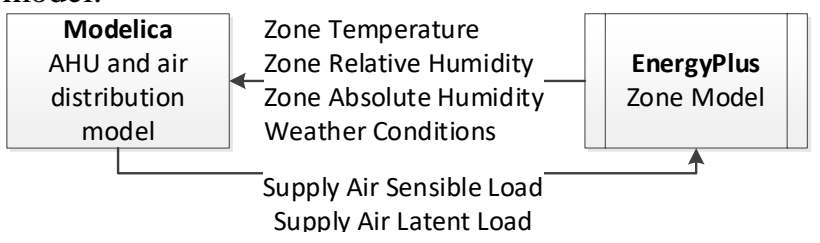

Figure 3. Modelica/EnergyPlus data exchange diagram.

\subsection{INDIGO demo site model}

To demonstrate the approach INDIGO has taken towards developing the models, part of a building that is supplied by a single air handling unit has been selected. This zone is called "Aislamiento" since it is the section where isolation rooms for immunodepressed patients are hospitalised. Hence, the Aislamiento zones are conditioned by a specific AHU because in those rooms the requested conditions are different. This AHU, which structure is identical to the one in Figure 2, supplied two zones that are kept at a pressure positive state. Figure 4 shows the main blocks of the model with corresponding variable exchange as built in Modelica.

\footnotetext{
${ }^{4}$ http://fmi-standard.org/
} 

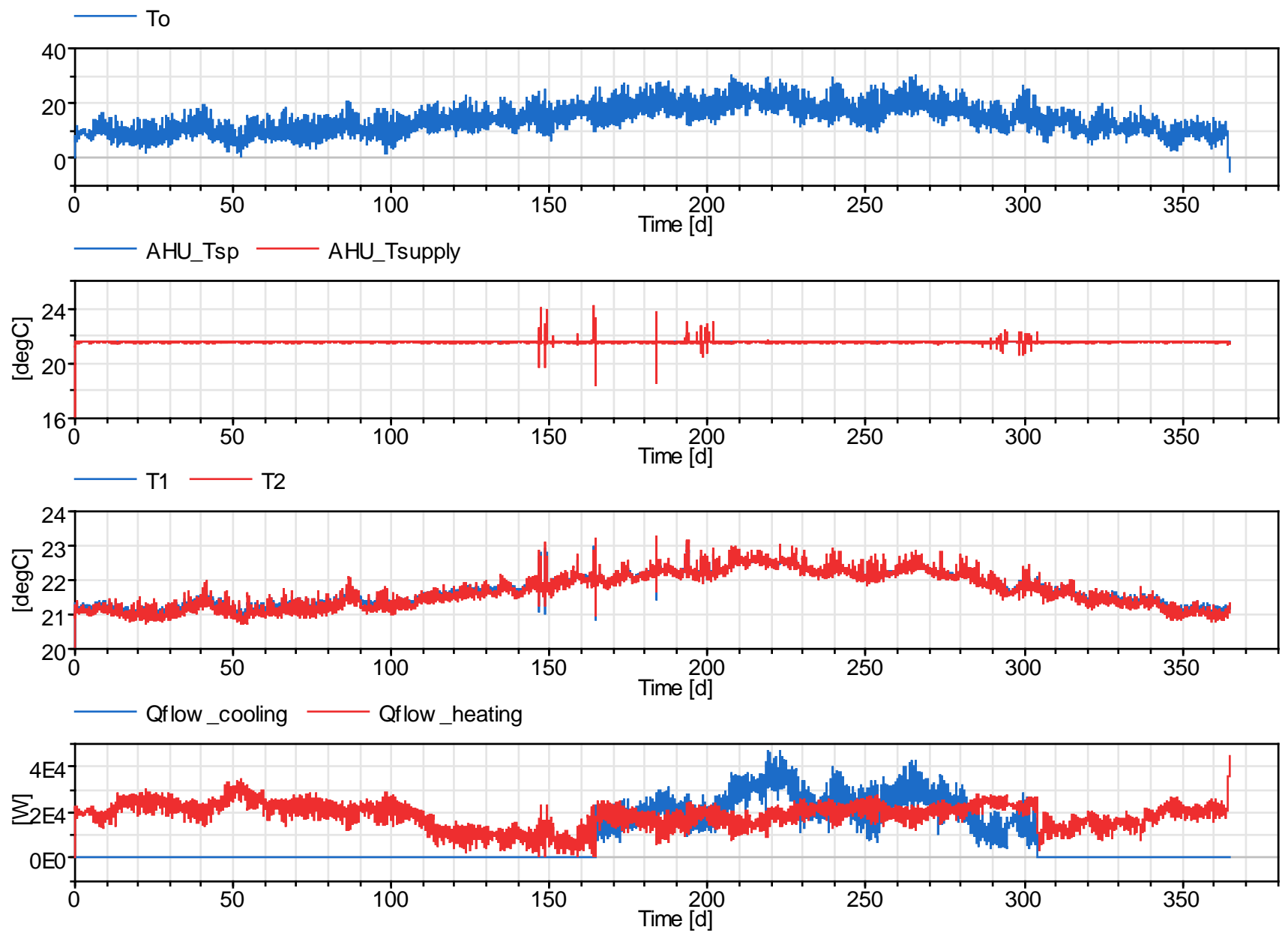

Figure 4. Results from 1-year simulation

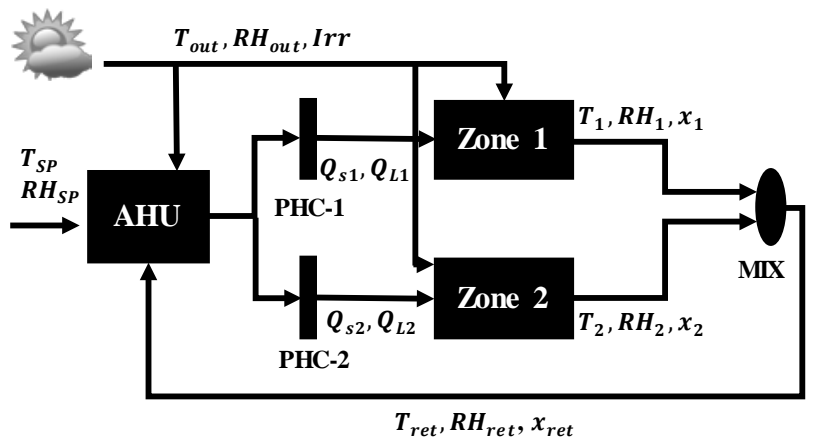

Figure 5. AHU Modelica Schematic

In Figure 4, T stands for Temperature, $\mathrm{RH}$ for relative humidity, x for absolute humidity, Qs for sensible heat, Ql for latent heat, PHC for post-heating coil and SP for setpoint. As mentioned, the model takes as inputs weather data and set-points and exchanges with the EnergyPlus FMU sensible and latent loads calculated also using the FMU Zone's information for indoor air conditions.

To use this model in the MPC developments, the whole building (AHU in Modelica and physical model in EnergyPlus-FMU) model was packaged in a FMU as shown in Figure 6.
From the whole building FMU (Figure 6), the model only needs as inputs weather data and outputs all the necessary variables to produce data for developing and training the MPC controllers. This includes not only the environmental conditions of the air in different point of the energy path but also the energy consumption of relevant elements such as coils.

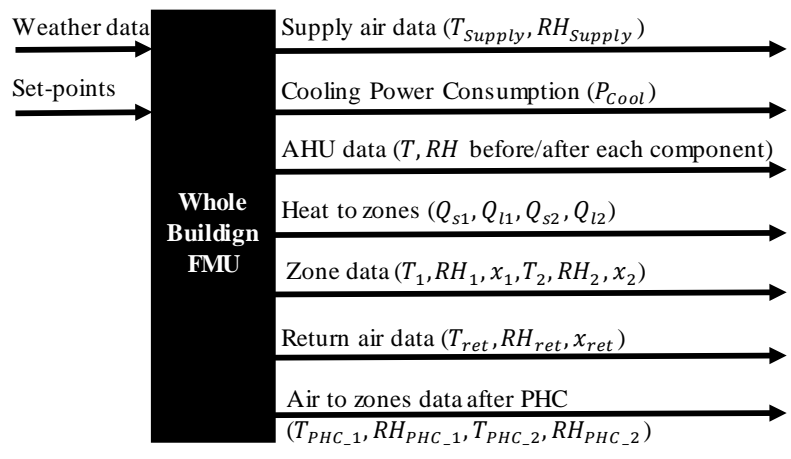

Figure 6. Whole Building FMU Schematic

\subsection{Model simulation}

Results from simulating the model for 1-year are presented in Figure 5. The purpose of performing the simulation was to validate the suitability of the model for use in MPC, to validate the values provided in the 
results were coherent, to check correct the integration of the different simulation tools via FMU and to check the proper implementation of the low-level controls. The model behaves as expected albeit some spikes appear that show in the middle of the year simulation which are caused by the mode-changing (e.g. state machine) implementation of the control system. However, such spikes do not affect the overall behaviour and result from the model.

\section{Interfacing for MPC and results}

This section provides details on the development of the Model Predictive Controller for the Air Handling Unit based on the FMU described in the previous section.

\subsection{Interfacing scheme}

Figure 7 shows the general interfacing scheme between the whole building models and the MPC development framework.

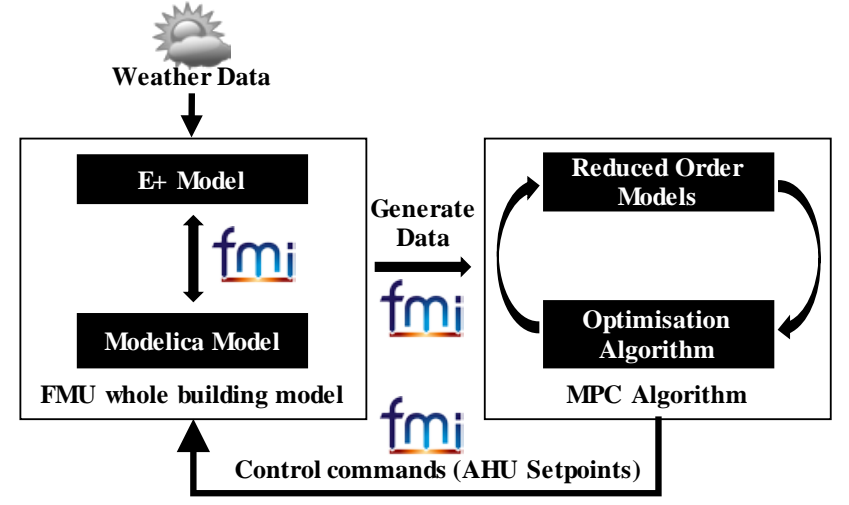

Figure 7 MPC development platform

The main purpose of the whole building models is to generate synthetic data to train the MPC algorithm on one side and on the other side to be used as a test-bed to check that the developed MPC performs as expected. The MPC algorithm development is explained in the following sections.

\subsection{Design of the MPC}

The developed MPC aims at minimising the energy consumption at building level while maintaining thermal comfort. The MPC is based on iterative, finitehorizon, optimisation of the objective function based on the dynamic model of the plant. The optimization is defined over the interval $[k, k+H]$, where $k$ is the current time and $H$ is the prediction (optimisation) horizon. Typically, only the first (discrete time) step of the solution is implemented, then the plant state is sampled again, and a new optimization is repeated in a receding horizon fashion (see Figure 8).

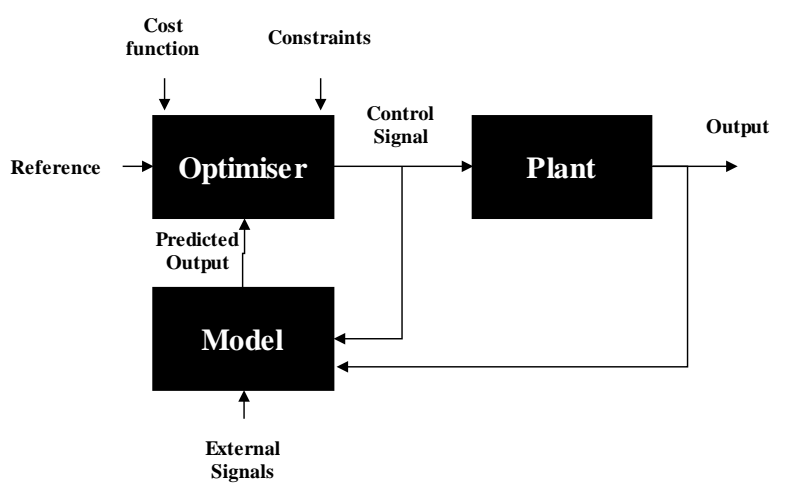

Figure 8. General diagram of a MPC.

The optimisation problem used by the proposed MPC is the following:

subject to constraints:

$$
\begin{aligned}
\min _{x_{t}, x_{r h}} \sum_{k=1}^{H}\left[w_{c}(k)\right. & \left.p_{c}(k)+w_{h}(k) p_{h}(k)\right] \\
& +\lambda \sum_{k=1}^{H}\left\{w_{t}\left[t_{r}(k)-t_{i}(k)\right]^{2}\right. \\
& \left.+w_{r}\left[r h_{r}(k)-r h_{i}(k)\right]^{2}\right\}
\end{aligned}
$$

$$
\begin{array}{cc}
\left(t_{r}, r h_{r}, p_{c}, p_{h}\right)=f\left(x_{t}, x_{r h}, \Theta\right) & 2 \\
x_{t, \min } \leq x_{t} \leq x_{t, \max } & 3 \\
x_{r h, \min } \leq x_{r h} \leq x_{r h, \max } & 4 \\
\left|x_{t}(k+1)-x_{t}(k)\right| \leq \rho, k=0, \ldots, H-1 & 5 \\
\left|x_{r h}(k+1)-x_{r h}(k)\right| \leq \gamma, k=0, \ldots, H-1 & 6 \\
t_{\min } \leq t_{r} \leq t_{\max } & 7 \\
r h_{\min } \leq r h_{r} \leq r h_{\max } & 8
\end{array}
$$

The optimization (control) variables are the supply temperature, $\boldsymbol{x}_{\boldsymbol{t}}$, and the supply relative humidity (RH), $\boldsymbol{x}_{\boldsymbol{r} \boldsymbol{h}}$, setpoints for the AHU. The function $\left(\boldsymbol{t}_{\boldsymbol{r}}, \boldsymbol{r} \boldsymbol{h}_{\boldsymbol{r}}, \boldsymbol{p}_{\boldsymbol{c}}, \boldsymbol{p}_{\boldsymbol{h}}\right)=f\left(\boldsymbol{x}_{\boldsymbol{t}}, \boldsymbol{x}_{\boldsymbol{r} \boldsymbol{h}}, \boldsymbol{\Theta}\right)$ is the building model that predicts the room temperature, room $\mathrm{RH}$ and the cooling and heating power as function of the control variables and the external inputs (predicted weather conditions and past room state). The problem computes the optimal setpoints for a prediction horizon of 24 hours. The objective function has two terms: 1) energy consumption and 2) deviation from desired comfort level. The regularization weight $\lambda$ is a positive constant that balances the trade-off between energy consumption and desired thermal comfort. The constraints impose lower and upper bounds for the supply AHU set-points and for the room temperature and RH. Smoothness constraints are also included to avoid abrupt changes in the setpoints in time.

We tested two types of reduced models for the MPC: 1) First principle based models and 2) long short-term memory recurrent neural network (LSTM-NN). The first-principle based model uses a simplified physical model for the AHU coupled with a linear autoregressive model for the room envelope. The coefficients of the auto-regressive model can be updated every week, or every season based on observed data. 
The LSTM-NN is a purely data-driven model that represents the whole system as an input-output function. The LSTM-NN is trained from simulated data (model from sections 3 and 4) using different weather profiles and setpoint strategies to avoid overfitting and achieve a good approximation of the system dynamics.

\subsection{Results from the MPC}

We tested the developed MPC in a simulation model of the building demo zone in the test site. The model consists of a room with an AHU. The MPC was tested using both the reduced physical models and NN models in the optimization for a period of 28 days in summer (June-July) with the constraints described in Table 2.

Table 2. Constraints imposed in the MPC problem.

\begin{tabular}{|c|c|c|c|}
\hline $\begin{array}{c}\text { Ideal room } \\
\text { temp. }\left(t_{i}\right)\end{array}$ & $21.5^{\circ} \mathrm{C}$ & $\begin{array}{c}\text { Ideal room } \\
\mathrm{RH}\left(r h_{i}\right)\end{array}$ & $50 \%$ \\
\hline $\begin{array}{c}\text { Max. room } \\
\text { temp. }\left(t_{\max }\right)\end{array}$ & $24^{\circ} \mathrm{C}$ & $\begin{array}{c}\text { Max. room } \\
\mathrm{RH}\left(r h_{\max }\right)\end{array}$ & $55 \%$ \\
\hline $\begin{array}{c}\text { Min. room } \\
\text { temp. }\left(t_{\min }\right)\end{array}$ & $20^{\circ} \mathrm{C}$ & $\begin{array}{c}\text { Min. room RH } \\
\left(r h_{\min }\right)\end{array}$ & $45 \%$ \\
\hline $\begin{array}{c}\text { Max. supply } \\
\text { temp. }\left(x_{t, \max }\right)\end{array}$ & $27^{\circ} \mathrm{C}$ & $\begin{array}{c}\text { Max. supply } \\
\mathrm{RH}\left(x_{r h, \max }\right)\end{array}$ & $65 \%$ \\
\hline $\begin{array}{c}\text { Min. supply } \\
\text { temp. }\left(x_{t, \min }\right)\end{array}$ & $21^{\circ} \mathrm{C}$ & $\begin{array}{c}\text { Min. supply } \\
\mathrm{RH}\left(x_{r h, \max }\right)\end{array}$ & $45 \%$ \\
\hline
\end{tabular}

The results are summarized in Table 3 also showing the results obtained using a standard PID controller for the room. The MPC is evaluated using two scenarios: a low comfort configuration with $\lambda=1$ and a higher comfort configuration with $\lambda=100$. The MPC coupled with the NN model and with $\lambda=1$ yield savings of approximately $62 \%$ in the cooling energy and $26 \%$ in the heating energy compared to the PID controller. However, it should be noted that the NN-based MPC achieves an average room temperature close to the ideal temperature but the room $\mathrm{RH}$ is far from the ideal. On the other hand, the results with $\lambda=100$ achieve room temperatures and $\mathrm{RH}$ close to the ideal ones at the expense of having a slightly larger energy consumption than the PID controller. In addition, it should be noted that the NN-based MPC are able to follow better the thermal comfort constraints than the Physical MPC. OIt is worth noticing that the $\mathrm{RH}$ has a big impact in the overall energy consumption of the building, thus, better strategies for RH control should be investigated, e.g. wider range for $\mathrm{RH}$.

\section{Conclusions}

This paper presented the developments of a detailed building energy model aimed at improving cooling control for further coupling with District Cooling.

\subsection{Modelica use}

In INDIGO, some advantages in the use of Modelica for modelling the energy systems where demonstrated:
Table 3. MPC results for 28 days between June and July using reduced Physical models (Ph-MPC) and NN models (NN-MPC).

\begin{tabular}{|l|c|c|c|c|c|}
\cline { 3 - 6 } \multicolumn{2}{c|}{} & \multicolumn{2}{c|}{$P h-M P C$} & \multicolumn{2}{c|}{$N N-M P C$} \\
\hline $\begin{array}{l}\text { Cooling energy } \\
\text { (kWh) }\end{array}$ & PID & $\begin{array}{l}\lambda \\
=1\end{array}$ & $\begin{array}{l}\lambda \\
=100\end{array}$ & $\begin{array}{l}\lambda \\
=1\end{array}$ & $\begin{array}{l}\lambda \\
=100\end{array}$ \\
\hline $\begin{array}{l}\text { Heating energy } \\
(\mathrm{kWh})\end{array}$ & 9,234 & 7,726 & 13,051 & 6,759 & 11,942 \\
\hline MAD temp. $\left({ }^{\circ} \mathrm{C}\right)$ & 0.16 & 2.27 & 2.01 & 1.13 & 1.16 \\
\hline Mean temp. $\left({ }^{\circ} \mathrm{C}\right)$ & 21.47 & 23.77 & 23.51 & 22.54 & 22.6 \\
\hline STD temp. $\left({ }^{\circ} \mathrm{C}\right)$ & 0.2 & 1.11 & 0.96 & 0.95 & 0.89 \\
\hline Min temp. $\left({ }^{\circ} \mathrm{C}\right)$ & 19.49 & 21.4 & 21.45 & 20.02 & 20.25 \\
\hline Max temp. $\left({ }^{\circ} \mathrm{C}\right)$ & 23.28 & 26.74 & 26.08 & 27.06 & 26.31 \\
\hline MAD RH $(\%)$ & 3.47 & 4.66 & 5.15 & 8.19 & 2.41 \\
\hline Mean $\mathrm{RH}(\%)$ & 52.31 & 53.98 & 45.09 & 57.23 & 48.57 \\
\hline STD RH $(\%)$ & 3.7 & 4.49 & 2.81 & 6.92 & 2.82 \\
\hline Min RH $(\%)$ & 20.96 & 0.05 & 4.69 & 39.37 & 0.06 \\
\hline Max RH $(\%)$ & 93.98 & 96.86 & 60.36 & 99.26 & 96.45 \\
\hline
\end{tabular}

- The hybrid modelling approach the Modelica enables in a single tool simplifies the modeler work, reduces error and provides an easier to use and understand approach to system's modelling. In Modelica, mechanical, electrical, and thermodynamic modelling can be integrated in the same model, including control algorithms;

- The object-oriented approach enables model reusability on the one side and on the other side, allows for modelling the physical systems following the physical structure as opposed to a signal structure used in other languages. This provides the clear advantage that models are easier to understand;

- The extension capabilities of Modelica via the Functional Mock-up Interface allowed to integrate models from different tools using an independent and standardized API into the MPC development environment, thus providing an integral solution for data analysis, simulation and optimization in one single environment.

\subsection{Functional Mock-up Interface use}

Given the variety of development tools used in INDIGO, to avoid the imposition of a single tool, which would have limited developments and to allow a seamless integration of the different developments, the use of Functional Mock-up units was agreed since all development tools were found to be compatible with the FMI standard. Embarking in such approach provided several benefits but also some challenges for INDIGO development which are described in the following sections. 


\subsubsection{Benefits}

Amongst the benefit of using the FMI standard we found:

- FMI is a standardised approach. For developers this means there is less effort in the integration between tools. They only need to agree on the variables to be exchanged as opposed to have to develop the integration interface itself.

- The FMI is tool-independent which translates in a seamless model exchange across different tools

- Models can be re-used for different purposes. This is a combined advantage between the use of Modelica and the use of FMI. All public variables can be made accessible by the FMU which means that there is no need to change the model in case a new variable needs to be exported. This results in less effort and better quality of the developments produced with FMI and Modelica based models.

\subsubsection{Challenges}

In INDIGO, the use of the FMI standard also exposed some challenges:

- Ensuring the efficiency and robustness of the models is fundamental for the FMUs generated in terms of usability, performance and error-handling when running simulations and generating data;

- While the standard for data exchange is certainly excellent, the integration over two platforms presents another challenge in the standardisation of the data to be exchanged.

- Different simulation tools might provide different results mainly due to the use of different solvers. This is something that needs to be acknowledged since it is, for the time being and for a typical modeller, not trivial;

- The parametrization of the models is not necessarily evident when doing model exchange, thus it requires modeller's attention;

- Documentation of the FMU needs to be provided separately from the model. If the modeller is used to Modelica where the documentation is contained within the model, this might be overlooked when exchanging the models

\subsection{MPC implementation}

MPC is a powerful control strategy that anticipates to future events and takes control actions accordingly. However, in order to achieve real-time control, the optimization problem has to be solved faster than the sampling time of the system. Thus, the reduced models used within the MPC are of great importance. On one hand, the model needs to be sufficiently simple and fast to be used in the optimization loop, and on the other hand, the model needs to be accurate enough to avoid erroneous control strategies due to approximation errors by the reduced models.

In INDIGO, we have explored the use of $\mathrm{NN}$ as reduced models for MPC with satisfactory results. The advantages of NN, especially recurrent NN such as the LSTM-NN, are twofold: firstly, fast computation time to allow its use within the MPC, and secondly, high accuracy in the modelling to capture both slow and rapid dynamics of the system. However, in order to capture the correct dynamic behaviour of the system, the NN has to be trained with data that explore a large portion of the data space and model dynamics, which is not often the case with data collected from a real site. Therefore, simulation platforms, such as the one developed in INIDIGO, are a great tool to generate training data for NN models within a MPC.

\section{Acknowledgments}

Project INDIGO has received funding from European Union's Horizon 2020 research and innovation programme under grant agreement $n^{\circ}$ 696098.

\section{References}

del Hoyo Arce, I. et al. (2018) 'Models for fast modelling of district heating and cooling networks', Renewable and Sustainable Energy Reviews. Elsevier Ltd, 82(June), pp. 1863-1873. doi: 10.1016/j.rser.2017.06.109.

Nouidui, T. S., Wetter, M. and Zuo, W. (2014) 'Functional mock-up unit for co-simulation import in \{EnergyPlus\}', Journal of Building Performance Simulation. Taylor \& Francis, 7(3), pp. 192-202. doi: 10.1080/19401493.2013.808265.

Reindl, D. T., Beckman, W. A. and Duffie, J. A. (1990) 'Diffuse fraction correlations', Solar Energy, 45(1), pp. 17. doi: https://doi.org/10.1016/0038-092X(90)90060-P.

Sterling, R. et al. (2017) 'Demand side detailed models'. doi: 10.5281/ZENODO.1137755. 